\title{
Validez y confiabilidad de una escala de medición del capital intelectual en PyMEs
}

\section{Validity and reliability of a scale for measuring intellectual capital in SMEs}

\author{
Omar Arodi Flores Laguna, Karla Saraí Basurto Gutiérrez*, \\ José Antonio Sánchez Valdez
}

Universidad de Montemorelos y Tecnológico Nacional de México, México

Recibido el 12 de enero de 2019; aceptado el 15 de octubre de 2019

Disponible en Internet el: 21 de octubre de 2019

\section{Resumen}

Esta investigación tuvo el propósito de realizar el análisis exploratorio, así como su confiabilidad de la Escala de Capital Intelectual (ECI) en las pequeñas y medianas empresas (pymes) de Monterrey, Nuevo León, México. La muestra estuvo constituida por 233 directivos de Pymes de Monterrey. Se validó un instrumento con 16 declaraciones para medir la variable capital intelectual en las pymes. Se realizó un análisis factorial exploratorio usando dos métodos, el de componentes principales y modelo de ecuaciones estructurales. Al realizar el análisis exploratorio del constructo se observó que los ítems se agruparon en los tres factores (capital humano, capital estructural y capital relacional) propuestos por diversos autores. Al realizar la validez del constructo usando modelos de ecuaciones estructurales se observó que todos los criterios seleccionados $\left(X^{2}, p, X^{2} / g l, C F I, G F I, N F I, R M S E A\right)$ se cumplieron, obteniéndose una alta confiabilidad de la escala.

Código JEL: M12, O10, O15

Palabras clave: Capital intelectual; Capital humano; Capital estructura; Capital relacional

* Autor para correspondencia

Correo electrónico kbasurto@um.edu.mx (K.S. Basurto Gutiérrez).

La revisión por pares es responsabilidad de la Universidad Nacional Autónoma de México. 


\begin{abstract}
This investigation had the purpose of doing the exploratory analysis of the intellectual capital (IC) construct and the reliability in small and medium enterprises (SME's) of Monterrey, Nuevo León, México. The sample used consisted of 233 SME's managers from Monterrey. A questioner with 16 statements was validated to measure IC in the SME's. The factorial analysis was made with two methods the principal components and structural equation modelling. It was observed that in the factorial analysis three factors got together (human capital, structural capital and relational capital) that were proposed by many authors. In the confirmatory analysis of the IC through structural equations, shows that all the criteria ( $\left.\mathrm{X}^{2}, \mathrm{p}, \mathrm{X}^{2} / \mathrm{gl}, \mathrm{CFI}, \mathrm{GFI}, \mathrm{NFI}, \mathrm{RMSEA}\right)$ have a good fit.
\end{abstract}

Código JEL: M12, O10, O15

Palabras clave: Intellectual capital; Human capital; Structure capital; Relational capital

\title{
Introduction
}

Edvinsson y Malone (1999) mencionan que una organización es como un árbol en donde hay una parte visible (hojas, ramas y frutos) y otra que está oculta (raíces). Si solamente se preocupa por la parte visible y se olvidan las raíces, el árbol puede morir. Esto también es válido para las empresas: si solo se preocupa de los resultados financieros y se ignora los valores ocultos, la empresa no sobrevivirá en el largo plazo. En una economía basada en el conocimiento, el capital intelectual (CI) es reconocido como una fuente de crecimiento, innovación y ventaja competitiva (Lev, 2001). Asimismo, gran parte de la literatura enfatiza el potencial del CI para mejorar la competitividad de una empresa, posición y creación de valor. También sugieren beneficios para superar las debilidades que como pequeñas y medianas empresas tienen (Berezinets, Garanina y Ilina, 2016; Edvinsson y Malone, 1997; Firer, 2005; Jordão y Novas, 2017; Sullivan, 2000; Verbano y Crema, 2016). El tema del CI ha ganado una consideración relevante entre académicos, profesionales y consultores; las empresas compiten confiando más en recursos intangibles como tecnologías, innovaciones en procesos, habilidades de los empleados, creatividad, relaciones con socios externos y redes industriales (Berezinets, et al. 2016; Cordazzo, 2005; Keong, 2008; Kujansivu y Lönnqvist, 2007).

Varios autores (Dumay, 2014; Galabova, 2014; Mouritsen, 2006) mencionan que a pesar de que el CI no se puede ver en un informe contable, predominan artículos con este punto de vista e ignoran procesos importantes de la gestión y la estrategia en la organización. Por otro lado, algunos eruditos señalan que las pymes no parecen administrar el CI como lo hacen las 
grandes empresas e instan al futuro investigador a hacer investigación del CI en las pymes (Durst y Edvardsson, 2012; Guthrie, Ricceri y Dumay, 2012; Marzo y Scarpino, 2016).

Este artículo tiene como objetivo principal proporcionar una escala de medición validada para medir el CI.

Esta investigación está dividida en las siguientes secciones: marco teórico, metodología, análisis de resultados, discusión, conclusiones y referencias.

\section{Marco teórico}

\section{Definiciones de capital intelectual}

¿Qué es el capital intelectual? Algunos autores lo definen como los activos intangibles que tiene una organización, pero que no están reflejados en los estados contables y que genera un valor o tiene potencial de generarlo en el futuro (Euroforum, 1998; Lev, 2001; Ramírez y Constansa, 2009; Sagástegui, 2014).

Según Medina, González y Pérez (2007), el CI incluye el conocimiento del personal, la capacidad para aprender y adaptarse, las relaciones con los clientes y los proveedores, las marcas, los nombres de los productos, los procesos internos y la capacidad de innovación y desarrollo. Para Secundo, Dumay, Schiuma y Passiante (2016), el CI es un concepto multidimensional de los activos de conocimiento, experiencia y capacidades prácticas para crear valor. Edvinsson y Malone (2003) mencionan que algunos autores incluyen en su definición de CI factores como el liderazgo en tecnología, formación de los empleados y aspectos como la rapidez de respuesta a los clientes. Cassol, Reis, Santos y Lima (2016) afirman que para el desarrollo del CI y para la capacidad de absorción e innovación, se necesita la capacitación constante de los empleados, los programas de sugerencias, la asimilación de nuevas tecnologías, la aplicación de los conocimientos técnicos y las asociaciones con organismos de apoyo a la innovación.

\section{Dimensiones}

La mayoría de los autores (Alvarez y Gonzáles, 2013; Bontis, 2002; Bontis, Chua y Richardson, 2000; Buenechea, 2017; Herremans, Isaac, Kline y Nazari, 2011; Marzo y Scarpino, 2016; Matricano, 2016; Mention y Bontis, 2013; Ordóñez, 2003; Petty y Guthrie, 2000; Reyes, 2011; Roos, Roos, Dragonetti y Edvinsson, 2001; Sagástegui, 2014; Viedma, 2001; Wee y Chua, 
2016) concuerdan en que las dimensiones del CI, desde su inicio, siguen clasificándose en las mismas tres dimensiones: (a) capital humano, (b) capital estructural y (c) capital relacional.

\section{Capital humano}

Para Mertens (1996), los directivos de las organizaciones le están dando mayor importancia al factor humano. La Unión Europea reconoce que las innovaciones y el factor humano pueden verse como los principales impulsores del crecimiento de los países, empresas y personas (OECD, 2013). El factor humano está presente en todos los momentos del hecho productivo, ya que se requiere de la participación de las personas para obtener resultados en el desempeño organizativo (Kemppilä y Lönnqvist, 2003; Saari y Judge, 2004).

Por otro lado, Reyes (2011) menciona que el capital humano es el talento que tienen las personas y es el principal valor que tienen las organizaciones. Para Marimuthu, Arokiasamy, e Ismail (2009), el capital humano es una de las dimensiones del CI conocida como la más grande y el activo intangible más importante de la organización, ya que incluye procesos asociados con educación, capacitación y otros planes de la carrera para aumentar el nivel de conocimientos, habilidades, valores y activos sociales de un empleado. Del mismo modo, otros autores señalan que el capital humano es la fuente de conocimiento, ideas, inspiración e innovación en las organizaciones (Gates y Langevin, 2010; Johanson, Mårtensson y Skoog, 1999).

Diversos autores mencionan que el capital humano es la suma de conocimientos, habilidades, competencias, innovaciones, compromiso y sabiduría que pertenecen al empleado y que usa para llevar acabo sus tareas y, que se lleva, al dejar la organización (Bontis, 1998; Edvinsson y Malone, 2003; Johnson, 1999; Morris, 2015).

El capital humano es el conjunto de competencias de un individuo que le permite generar valor para la empresa. Este se compone de entrenamiento, incentivos, trabajo en equipo, reconocimiento público, premios, ascensos, cumplimiento de metas, rotación de trabajadores e información suficiente y cierta (Roos et al., 2001; Sagástegui, 2014). Por su parte, Álvarez y Gonzáles (2013) mencionan que el capital humano incluye la parte emocional y la operativa, con sus actitudes, sentimientos, motivación, lealtad, amistad y conducta. Sagástegui (2014) señala que se pueden distinguir tres componentes dentro de la dimensión dedicada al capital humano: (a) las competencias, en forma de conocimientos, capacidades y talento y saber cómo hacerlo; (b) la actitud, que se traduce en conducta, motivación, actuación y ética de las personas; y (c) la agilidad intelectual, la cual genera valor para la organización en la medida en que se aplican conocimientos nuevos o descubrimientos que permiten transformar las ideas en productos y servicios. 


\section{Capital estructural}

El capital estructural es más especializado que los otros componentes, ya que puede ser visto como la estructura básica de una empresa que potencia al capital humano. Además, el capital estructural se considera como la estructura para el establecimiento y mantenimiento de las relaciones en la organización. Así mismo, el capital estructural comprende los activos estratégicos más valiosos de la empresa, como capacidades organizacionales, culturales, procesos, patentes, derechos de autor, marcas registradas y bases de datos (Bontis, 1998; Curado, Henriques y Bontis, 2011; Denicolai, Ramusino y Sotti, 2015; Hejazi, Ghanbari y Alipour, 2016; Janosevic y Dzenopoljac, 2012; Johnson, 1999; Molodchik, Shakina y Barajas, 2014; Schiuma y Lerro, 2008).

Para Aramburu, Sáenz y Blanco (2015), el capital estructural explica en gran medida la efectividad del proceso de generación de nuevas ideas y de la gestión de proyectos de innovación, a causa de que el capital estructural incluye equipos, programas, bases de datos, estructura organizacional y todo lo que forma parte de la capacidad organizacional; es decir, lo que se queda en la empresa cuando los empleados no están en el trabajo (Edvinsson y Malone, 2003).

Por su parte, Medina et al. (2007) mencionan que hay dos posiciones de pensamiento para medir el capital estructural. La primera proviene de la protección legal que la organización ejercita sobre aquellos activos que tienen un valor especial para la misma, entre los que se encuentran el saber cómo, los secretos de fabricación, el copyright, las patentes, los derechos de diseños y las marcas de fábrica y de servicios. La segunda hace referencia a los activos que aportan orden, seguridad, corrección, calidad a la organización, la cultura corporativa y los métodos para el cálculo de riesgos o las bases de datos de información

Por otro lado, Sagástegui (2014) menciona que el capital estructural se refiere a la estructura organizacional formal e informal y a los sistemas de dirección, gestión y cultural de la empresa. El capital estructural tiene que ver con diseño estructural, mecanismos de coordinación, comportamiento grupal, rutinas organizativas, cultura corporativa, sistemas de planificación y control y conocimientos de carácter técnico industrial.

\section{Capital relacional}

El capital relacional es el conocimiento obtenido a través del desarrollo de relaciones externas interesadas. Este comprende conocimiento de los empleados, procesos, capacidad de innovación, proyectos de investigación, marca y relaciones (Bontis, Janosevic y Dzenopoljac, 
2015; Cabrita y Bontis, 2008; Johnson, 1999; Kweh, Lu y Wang, 2014; Serenko y Bontis, 2004; Ting y Lean, 2009; Wang, Wang y Liang, 2014; Youndt, Subramaniam y Snell, 2004; Yu, Wang y Chang, 2015;).

Para Edvinsson y Malone (1997), el capital relacional incluye relaciones externas como por ejemplo con clientes, proveedores, accionistas y otras partes interesadas como el gobierno y sociedad en general. Sagástegui (2014) menciona que el capital relacional está compuesto por las relaciones que tiene la empresa con sus clientes (precio y control de calidad), con canales de distribución, proveedores, alianzas, bancos, accionistas, marcas comerciales; por lo tanto, este capital tiene que ver con las relaciones que tiene la empresa. Visto de otra manera, es la percepción de valor que se tiene sobre la empresa.

Por su parte, Álvarez y Gonzáles (2013) dividen el capital relacional en agentes externos e internos. Los agentes externos son los clientes, proveedores, vendedores y administraciones públicas. Los agentes internos tienen que ver con las buenas relaciones con los accionistas, directivos, trabajadores, mercado (imagen y logotipo), reputación, ética y marca.

Para Villena y Souto (2015), tanto el capital relacional orientado al comercio exterior como la cultura sostenible de la empresa, la certificación de calidad, la capacidad de mejora competitiva y la orientación hacia el empleado impactan de manera positiva en el desempeño de la empresa.

\section{Reactivos propuestos de acuerdo con los autores}

En la Tabla 1 se presenta la base bibliográfica de cada reactivo.

Tabla 1

Reactivos con base bibliográfica

\begin{tabular}{|c|c|}
\hline Reactivos & Autores \\
\hline $\begin{array}{l}\text { 1. El personal aprende continuamen- } \\
\text { te de otros. }\end{array}$ & $\begin{array}{l}\text { (Bontis, 1998; Edvinsson y Malone, 2003; Johnson, 1999; Marimuthu et } \\
\text { al., 2009; Morris, 2015) }\end{array}$ \\
\hline $\begin{array}{l}\text { 2. Los trabajadores cuentan con } \\
\text { competencias necesarias para el } \\
\text { puesto }\end{array}$ & $\begin{array}{l}\text { (Bontis, 1998; Edvinsson y Malone, 2003; Johnson, 1999; Marimuthu et } \\
\text { al., 2009; Morris, 2015) }\end{array}$ \\
\hline $\begin{array}{l}\text { 3. El trabajador participa en los pro- } \\
\text { gramas de enriquecimiento de } \\
\text { puesto y flexibilidad laboral. }\end{array}$ & $\begin{array}{l}\text { (Bontis, 1998; Edvinsson y Malone, 2003; Johnson, 1999; Marimuthu et } \\
\text { al., 2009; Morris, 2015) }\end{array}$ \\
\hline $\begin{array}{l}\text { 4. El empleado es capaz de desarro- } \\
\text { llar nuevas ideas y conocimiento. }\end{array}$ & (Gates y Langevin, 2010; Johanson et al., 1999) \\
\hline
\end{tabular}


5. El personal hace frente a las dificultades con eficiencia

6 . Fomenta la confianza y se encuentra comprometido con la empresa.

7. Se cuenta con programas de entrenamiento para desarrollar y actualizar las competencias de los empleados constantemente.

8. Tienen procesos de reclutamiento y selección para contratar a los mejores candidatos

9. Se almacena el conocimiento y experiencias de los empleados en manuales, sistemas y procesos.

10. Tienen procedimientos documentados que ayudan a ejecutar acciones de rutina.

11. Cuentan con la infraestructura para que los empleados accedan a información relevante.

12. Evalúa de manera formal las razones de éxito o fracaso de la colaboración con las alianzas estratégicas.

13. Los empleados cuentan con buenas relaciones a través de redes de trabajo con clientes, proveedores, socios y amigos para el desarrollo de soluciones.

14. Se aprende de las alianzas estratégicas para mejorar sus procesos.

15. Los empleados se apoyan en los proveedores en la solución de necesidades empresariales y estratégicas (costos, calidad, tiempos, producción etc.).
(Bontis, 1998; Johnson, 1999; Edvinsson y Malone, 2003; Morris, 2015)

(Roos et al., 2001; Sagástegui, 2014)

(Bontis, 1998; Edvinsson y Malone, 2003; Johnson, 1999; Marimuthu et al., 2009; Morris, 2015)

(Bontis, 1998; Curado et al., 2011; Denicolai et al., 2015; Hejazi et al., 2016; Janosevic y Dzenopoljac, 2012; Johnson, 1999; Molodchik et al., 2014; Sagástegui, 2014; Schiuma y Lerro, 2008;)

(Bontis 1998; Johnson, 1999; Curado et al., 2011; Denicolai et al., 2015; Hejazi et al., 2016; Janosevic y Dzenopoljac, 2012; Molodchik et al., 2014; Sagástegui, 2014; Schiuma y Lerro, 2008)

(Bontis 1998; Curado et al., 2011; Denicolai et al., 2015; Johnson, 1999; Hejazi et al., 2016; Janosevic y Dzenopoljac, 2012; Molodchik et al., 2014; Sagástegui, 2014; Schiuma y Lerro, 2008)

(Bontis 1998; Curado et al., 2011; Denicolai et al., 2015; Janosevic y Dzenopoljac, 2012; Johnson, 1999; Molodchik et al., 2014; Sagástegui, 2014; Schiuma y Lerro, 2008)

(Bontis 1998; Johnson, 1999; Schiuma y Lerro, 2008; Curado et al., 2011; Denicolai et al., 2015; Hejazi et al., 2016; Janosevic y Dzenopoljac, 2012; Molodchik et al., 2014; Sagástegui, 2014)

(Bontis et al., 2015; Cabrita y Bontis, 2008; Edvinsson y Malone, 1997; Johnson, 1999; Kweh et al., 2014; Sagástegui, 2014; Serenko y Bontis, 2004; Ting y Lean, 2009; Wang et al., 2014; Youndt et al., 2004; Yu et al., 2015)

(Bontis et al., 2015; Cabrita y Bontis, 2008; Edvinsson y Malone, 1997; Johnson, 1999; Kweh et al., 2014; Sagástegui, 2014; Serenko y Bontis, 2004; Ting y Lean, 2009; Wang et al., 2014; Youndt et al., 2004; Yu et al., 2015;)

(Bontis et al., 2015; Cabrita y Bontis, 2008; Edvinsson y Malone, 1997; Johnson, 1999; Kweh et al., 2014; Sagástegui, 2014; Serenko y Bontis, 2004; Ting y Lean, 2009; Wang et al., 2014; Youndt et al., 2004; Yu et al., 2015;) 
16. El personal se ocupa de gestionar relaciones a largo plazo con los clientes.
(Bontis et al., 2015; Cabrita y Bontis, 2008; Edvinsson y Malone, 1997; Johnson, 1999; Kweh et al., 2014; Sagástegui, 2014; Serenko y Bontis, 2004; Ting y Lean, 2009; Wang et al., 2014; Youndt et al., 2004; Yu et al., 2015;)

Fuente: Elaboración propia

\section{Investigaciones sobre la medición de la escala del CI}

Martínez (2006) realizó un estudio exploratorio de un modelo de CI usando ecuaciones estructurales. El estudio se realizó en una universidad en el área de humanidades y ciencias sociales. El tamaño de la muestra fue de 59 sujetos. El instrumento se conformó con 60 reactivos los cuales fueron clasificados en 10 mapas conceptuales. El constructo CI fue dimensionado en capital humano, capital estructural y capital relacional. La confiabilidad del instrumento para capital humano fue de .81, para capital estructural .72 y para capital relacional .82. Además, probaron las siguientes hipótesis: (a) el capital humano predice al capital estructural en un $29 \%$, (b) el capital estructural predice al capital relacional en un 50\% y (c) capital relacional predice al capital humano en un $36 \%$.

Cabrita y Bontis (2008) realizaron un estudio del CI y el desempeño de la industria bancaria portuguesa. El estudio se realizó con 253 participantes de 53 organizaciones bancarias. El instrumento de CI se dimensionó en capital humano, capital relacional y capital estructural. Además, se midió el desempeño de los negocios. Las hipótesis se probaron utilizando el software PLS. La confiabilidad de los instrumentos fue de .93 . Se realizó un análisis de componentes principales con una rotación VARIMAX, donde los cuatro factores quedaron conformados por 14 ítems para capital humano, 14 para capital relacional, 10 para capital estructural y 10 para medir el desempeño. Posteriormente se realizó una comparación con estudios de Canadá y Malasia donde se encontró que 15 ítems se repiten en los tres estudios.

Hernández, Moreno y Arroyo (2010) realizaron un análisis exploratorio de la implementación y el uso de los sistemas de información sobre el CI en las empresas. El proceso de recolección de datos fue por medio de un instrumento que enviaron a los directores o al departamento de control de gestión de las 1,911 empresas de la Comunidad Autónoma de Castilla y León. El índice de respuesta fue del 11\% y la muestra fue de 211 empresas. Para medir el CI utilizaron las siguientes dimensiones: capital humano, capital estructural y capital relacional.

Chahal y Bakshi (2016) realizaron una investigación para medir el CI en el sector bancario. El instrumento fue distribuido a 576 participantes de 144 sucursales, de los cuales 339 respondieron el cuestionario. La confiabilidad del instrumento total de CI fue de .922; del capital humano .913 , del capital estructural .820 y del capital relacional .908 . En el análisis 
exploratorio, en la dimensión de capital humano, con 31 ítems se redujo a 13. En el capital estructural se retuvieron 16 de 21 ítems y en el capital relacional, de los 11 ítems se retuvieron siete. En seguida realizaron un análisis confirmatorio usando los tres factores (capital humano, capital estructural y capital relacional). Estos tres factores quedaron dimensionados de la siguiente manera: (a) capital humano: competencias, creatividad, actitud del administrador y actitud del empleado, (b) capital estructural: innovación, tecnologías de la información y cultura y (c) capital relacional: relación con los empleados y relación con los clientes. Los resultados obtenidos del modelo fueron los siguientes: $X^{2} / g l$ igual a $3.119, R M S E A$ igual a $.80, N F I$ igual a .914, IFI igual a $.940, T L I$ igual a .912 y $C F I$ igual a .939 .

Mercado (2016) realizó una investigación con respecto a la validez de una escala de medición del capital intelectual, con un modelo de tres componentes (capital humano, capital estructural y capital relacional). La validación se realizó con una muestra de 742 académicos, mandos medios y directivos de universidades mexicanas. En el análisis factorial exploratorio se identificaron cuatro factores (capital humano, capital estructural, capital relacional y capital tecnológico) que explican el 66.74 de la varianza. La confiabilidad extraída por el alfa de Cronbach fue mayor a .85 .

\section{Metodología}

La población utilizada en esta investigación estuvo formada por los directivos de 2500 Pymes registradas en CAINTRA en el estado de Nuevo León, México. Para el proceso de recolección de la muestra se solicitó la autorización del director del Tecnológico Nacional de México, campus Nuevo León, mediante un oficio firmado por el jefe de proyecto de investigación y el de vinculación de proyectos de ingeniería industrial. Este oficio turnó la petición del permiso a los directores de las empresas afiliadas a CAINTRA. Posteriormente, a un grupo de estudiantes que realizaba las residencias profesionales en estas empresas se le proporcionó un número de 10 encuestas a cada uno, junto con el oficio para ser presentado a los directivos de las empresas y que, así, dieran autorización para la aplicación de la encuesta. La encuesta se aplicó de forma física a los directivos en sus instalaciones, en su tiempo libre; para otras se agendaron citas, con el fin de no obstruir las labores productivas diarias de las empresas.

El tipo de muestreo realizado en esta investigación fue no aleatorio y por conveniencia, ya que se seleccionó a los directivos de las empresas de Monterrey, Nuevo León, México y su área metropolitana, que estuvieron dispuestos a participar entre los meses de septiembre a diciembre del año 2017. La muestra fue de 233 directivos, que representan el $9.32 \%$ de la población y tiene las siguientes características: (a) en relación con la edad, de 20 a 30 años, el $48.7 \%$; de 31 a 40, el $29.3 \%$; de 41 a 50 , el $18.5 \%$ y de 51 a 60 , el $3.4 \%$; (b) con respecto 
al género, el $79.3 \%$ son hombres y el $20.7 \%$, mujeres; (c) sobre el nivel académico, el 14.2\% estudiaron hasta preparatoria; el 69.4\%, hasta licenciatura y, hasta el posgrado, solo el 16.4\%; (d) en relación a su área de desempeño, el 3.9\% lo hace en ventas; el 34.3\%, en pro-ducción; el $8.2 \%$, en compras; el 12.4\%, en administración y, en otras áreas, el $41.2 \%$; (e) con respecto al puesto que tienen, el $17 \%$ trabaja en el nivel operativo; el 59.2\% es supervisor; el $22.9 \%$ de ellos trabajan en la gerencia y, en la dirección, solo el .9\%; (f) en relación al sector, el $4.8 \%$ está en ventas; en fabricación, el $88.3 \%$ y, en servicios, el $6.9 \%$.

\section{Procedimiento en la elaboración del instrumento}

Sánchez (2018) elaboró un instrumento con 16 declaraciones de Escala de Capital Intelectual (ECI) que quedó conformado por tres dimensiones, así como lo sugieren los autores mencionados anteriormente: (a) capital humano (CI1 a CI7), (b) capital estructural (CI8 a CI11) y (c) capital relacionado a la organización (CI12 a CI16). Con respecto al procedimiento, se realizó un listado de ítems que se podrían utilizar para formar el constructo; en seguida, se realizaron consultas y revisiones con expertos en el tema como directores de pymes para seleccionar los ítems más pertinentes, quedando un total de 16 ítems.

Después de conformar el instrumento, se aplicó la validez de contenido donde se evaluaron la claridad y la pertinencia de cada uno de los ítems con la ayuda de cinco expertos en la temática. El instrumento fue aplicado entre los meses de septiembre a diciembre del año 2017. La escala utilizada fue la siguiente: 1 , nunca; 2 , casi nunca; 3 , a veces; 4, casi siempre y 5 , siempre.

Hair, Anderson, Tatham y Black (2007) sugieren tener por lo menos cinco encuestados por cada ítem. El ratio utilizado en esta investigación fue de 15 encuestados por cada reactivo.

\section{Análisis de resultados}

Para determinar la validez del constructo se realizó un análisis factorial exploratorio utilizando el software SPSS versión 23. Se calculó la confiabilidad del instrumento y se presentaron los estadísticos descriptivos. También se efectuó el análisis con modelos de ecuaciones estructurales utilizando el software AMOS versión 21. 


\section{Validez de constructo}

Se utilizó el procedimiento de análisis factorial para evaluar la validez del constructo CI. En el análisis de la matriz de correlaciones, se encontró que las 16 declaraciones tienen un coeficiente de correlación positivo mayor a .3 o muy próximo. En cuanto a la medida de adecuación muestral $K M O$, resultó un valor muy próximo a la unidad ( $K M O=.939)$. Para la prueba de esfericidad de Bartlett, se encontró que los resultados $\left(X^{2}=2,538.301, g l=120\right.$, $p=.000)$ son significativos. Al analizar la matriz de covarianza anti-imagen, se verificó que los valores de la diagonal principal son significativamente mayores a cero (mayores a .8).

Para el estadístico de extracción por componentes principales se encontró que los valores de comunalidad $\left(\mathrm{Com}_{\min }=.555 ; \mathrm{Com}_{\text {máx }}=.779\right)$ para los 16 reactivos de la escala son superiores al criterio de extracción $(\mathrm{Com}=.300)$. En relación con la varianza total explicada, se realizó un análisis confirmatorio con tres factores explicando un $69.143 \%$ de la varianza total, siendo este valor superior al $60 \%$ establecido como criterio.

En cuanto a la solución factorial rotada, se utilizó la rotación ortogonal. En la Tabla 2 se presenta información en donde se comparan las saturaciones relativas de cada reactivo para los tres factores de CI.

El primer factor (columna 1 de la Tabla 2) quedó constituido por seis ítems y se le asignó el nombre "capital humano". Los indicadores fueron los siguientes: "es capaz de desarrollar nuevas ideas y conocimiento" (CI4), hace frente a las dificultades con eficiencia" (CI5), "fomenta la confianza y se encuentra comprometido con la empresa" (CI6), "cuenta con competencias necesarias para el puesto" (CI2), "aprende continuamente de otros" (CI1), "es capaz de participar en los programas de enriquecimiento de puesto y flexibilidad laboral" (CI3) y "se cuenta con programas de entrenamiento para desarrollar y actualizar las competencias de los empleados constantemente" (CI7) a pesar de tener una carga factorial mayor en el factor tres (capital estructural) se decidió dejarlo en este factor (capital humano) puesto que tiene una carga importante en este factor y es en el que estaba inicialmente.

El segundo factor (columna 2 de la Tabla 2) quedó constituido por cinco ítems y se le asignó el nombre de "capital relacionado con la organización”. Los indicadores fueron los siguientes: "los empleados se apoyan en los proveedores en la solución de necesidades empresariales y estratégicas, costos, calidad, tiempos, producción, etc." (CI15), "aprende de las alianzas estratégicas para mejorar sus procesos" (CI14), "los empleados cuentan con buenas relaciones a través de redes de trabajo con clientes, proveedores, socios y amigos para el desarrollo de soluciones" (CI13), "el personal se ocupa de gestionar relaciones a largo plazo con los clientes" (CI16), el ítem CI12 (evalúa de manera formal las razones de éxito o fracaso 
de la colaboración con las alianzas estratégicas) a pesar de tener una carga factorial mayor en el factor tres (capital estructural), se decidió dejarlo en este factor (capital relacional) puesto que tiene una carga importante en este factor y es en el que estaba inicialmente.

El tercer factor (columna 3 de la Tabla 2) quedó constituido por cinco ítems y se le asignó el nombre de "capital estructural". Los indicadores fueron los siguientes: "tienen procedimientos documentados que ayudan a ejecutar acciones de rutina" (CI10), "almacenan el conocimiento y experiencias de los empleados en manuales, sistemas y procesos" (CI9), "cuentan con la infraestructura para que los empleados accedan a información relevante" (CI11) y "tienen procesos de reclutamiento y selección para contratar a los mejores candidatos" (CI8).

Tabla 2

Distribución de los reactivos después de una rotación ortogonal

\begin{tabular}{|c|c|c|c|}
\hline & \multicolumn{3}{|c|}{ Componente } \\
\hline & 1 & 2 & 3 \\
\hline Es capaz de desarrollar nuevas ideas y conocimiento (CI4) & .831 & .230 & .191 \\
\hline Hace frente a las dificultades con eficiencia (CI5) & .797 & .054 & .268 \\
\hline $\begin{array}{l}\text { Fomenta la confianza y se encuentra comprometido con la empresa } \\
\text { (CI6) }\end{array}$ & .724 & .284 & .296 \\
\hline Cuenta con competencias necesarias para el puesto (CI2) & .716 & .302 & .301 \\
\hline Aprende continuamente de otros (CI1) & .670 & .368 & .223 \\
\hline $\begin{array}{l}\text { Es capaz de participar en los programas de enriquecimiento de puesto y } \\
\text { flexibilidad laboral (CI3) }\end{array}$ & .578 & .474 & .346 \\
\hline $\begin{array}{l}\text { Los empleados se apoyan en los proveedores en la solución de necesi- } \\
\text { dades empresariales y estratégicas, costos, calidad, tiempos, producción } \\
\text { etc. (CI15) }\end{array}$ & .255 & .789 & .278 \\
\hline Aprende de las alianzas estratégicas para mejorar sus procesos (CI14) & .144 & .784 & .377 \\
\hline $\begin{array}{l}\text { Los empleados cuentan con buenas relaciones a través de redes de tra- } \\
\text { bajo con clientes, proveedores, socios y amigos para el desarrollo de } \\
\text { soluciones (CI13) }\end{array}$ & .282 & .769 & .218 \\
\hline $\begin{array}{l}\text { El personal se ocupa de gestionar relaciones a largo plazo con los clien- } \\
\text { tes (CI16) }\end{array}$ & .377 & .731 & .183 \\
\hline $\begin{array}{l}\text { Tienen procedimientos documentados que ayudan a ejecutar acciones } \\
\text { de rutina (CI10) }\end{array}$ & .243 & .250 & .749 \\
\hline
\end{tabular}




\begin{tabular}{|c|c|c|c|}
\hline $\begin{array}{l}\text { Almacenan el conocimiento y experiencias de los empleados en manua- } \\
\text { les, sistemas y procesos (CI9) }\end{array}$ & .365 & .201 & .726 \\
\hline $\begin{array}{l}\text { Cuentan con la infraestructura para que los empleados accedan a infor- } \\
\text { mación relevante (CI11) }\end{array}$ & .245 & .352 & .678 \\
\hline $\begin{array}{l}\text { Tienen procesos de reclutamiento y selección para contratar a los me- } \\
\text { jores candidatos }(\mathrm{CI} 8)\end{array}$ & .424 & .246 & .648 \\
\hline $\begin{array}{l}\text { Evalúa de manera formal las razones de éxito o fracaso de la colabora- } \\
\text { ción con las alianzas estratégicas (CI12) }\end{array}$ & .139 & .564 & .570 \\
\hline $\begin{array}{l}\text { Se cuenta con programas de entrenamiento para desarrollar y actualizar } \\
\text { las competencias de los empleados constantemente (CI7) }\end{array}$ & .452 & .368 & .464 \\
\hline
\end{tabular}

Fuente: Elaboración propia.

\section{Confiabilidad del instrumento}

El análisis se realizó con la escala completa, así como con cada una de las dimensiones propuestas; los resultados se presentan en la Tabla 3 . Se observan valores de confiabilidad muy aceptables. El del capital humano fue $(\alpha=.906)$ el valor más alto, mientras que el factor capital estructural ( $\alpha=.841)$ presentó la confiabilidad más baja, pero por encima del umbral $(\alpha=.700)$ que se tomó, y para capital relacional fue $(\alpha=.892)$. En general, el instrumento es confiable $(\alpha=.945)$.

Tabla 3

Valores de consistencia interna (alfa de Cronbach)

\begin{tabular}{lc}
\hline Escala & Alfa Cronbach \\
\hline Completa & .945 \\
Capital Humano & .906 \\
Capital estructural & .841 \\
Capital relacional & .892 \\
\hline
\end{tabular}

Fuente: elaboración propia.

En la Tabla 4 se muestran las medias aritméticas $(M)$ y la desviación estándar $(D E)$ del constructo CI. Las medias más altas fueron las siguientes: "Tiene procedimientos documentados que ayudan a ejecutar acciones de rutina" (4.25), "Fomenta la confianza y se encuentra comprometido con la empresa" (4.18) y "Cuentan con la infraestructura para que los em- 
pleados accedan a información relevante" (4.12). Las más bajas fueron las siguientes: "El trabajador participa en los programas de enriquecimiento de puesto y flexibilidad laboral" (3.68), "Se cuenta con programas de entrenamiento para desarrollar y actualizar las competencias de los empleados constantemente" (3.69) y "Evalúa de manera formal las razones de éxito o fracaso de la colaboración con las alianzas estratégicas" (3.77). La media total del constructo CI fue de 3.95 .

Tabla 4

Medias aritméticas de los ítems

\begin{tabular}{lll}
\hline Ítems & $M$ & $D E$ \\
\hline
\end{tabular}

Tienen procedimientos documentados que ayudan a ejecutar acciones de rutina (CI10).

Fomenta la confianza y se encuentra comprometido con la empresa (CI6).

Cuentan con la infraestructura para que los empleados accedan a información relevante (CI11).

El personal aprende continuamente de otros (CI1).

Los trabajadores cuentan con competencias necesarias para el puesto (CI2).

Tienen procesos de reclutamiento y selección para contratar a los mejores candidatos (CI8).

El empleado es capaz de desarrollar nuevas ideas y conocimiento (CI4).

El personal se ocupa de gestionar relaciones a largo plazo con los clientes (CI16).

El personal hace frente a las dificultades con eficiencia (CI5).

Se almacena el conocimiento y experiencias de los empleados en manuales, sistemas y procesos (CI9).

Los empleados cuentan con buenas relaciones a través de redes de trabajo con clientes, proveedores, socios y amigos para el desarrollo de soluciones (CI13). Los empleados se apoyan en los proveedores en la solución de necesidades empresariales y estratégicas (costos, calidad, tiempos, producción etc.) (CI15).

Se aprende de las alianzas estratégicas para mejorar sus procesos (CI14). 
Se cuenta con programas de entrenamiento para desarrollar y actualizar

las competencias de los empleados constantemente (CI7).

El trabajador participa en los programas de enriquecimiento de puesto y flexibilidad laboral (CI3).

Fuente: Elaboración propia.

Validez de constructo con ecuaciones estructurales

Para realizar el análisis factorial de ECI también se usó el modelo de ecuaciones estructurales. La base de datos en el software SPSS tenía varios datos perdidos y es necesario no tener datos perdidos para usar modelos de ecuaciones estructurales; se procedió a depurar la base de datos utilizando la media aritmética en cada uno de los ítems. Se construyó el diagrama del modelo donde se muestran las dimensiones y reactivos del constructo CI. En el modelo se establecieron las relaciones entre las dimensiones.

Hair et al. (2007) comentan que para evaluar el modelo se pueden utilizar los índices de bondad de ajuste (ver Tabla 4). De la lista fueron seleccionados algunos criterios que son los más utilizados, como son los siguientes: chi cuadrado $\left(X^{2}\right)$, razón chi cuadrado/grados de libertad $\left(X^{2} / g l\right)$, índice de bondad de ajuste comparativo $(C F I)$, índice de bondad de ajuste (GFI) y raíz de residuo cuadrático (RMSEA).

En la Figura 1 se observa el modelo propuesto para el CI, conformado por 16 ítems. Al resolver el modelo no se detectaron problemas, ya que las varianzas correspondientes a los ítems fueron positivas y los coeficientes estandarizados menores que uno. Examinando los coeficientes de regresión, se encontró que cada uno de ellos fue significativo a niveles de $\mathrm{p}$ menor o igual a .001 .

Para comenzar el proceso de validación del constructo CI, primero se revisó la normalidad del constructo, utilizando las distancias de Mahalanobis. Los datos se redujeron de 233 a 172. Los coeficientes de regresión estandarizados se revisaron y se ajustaron a 1. Posteriormente, se revisaron los momentos residuales, así como el ajuste de los índices, dando como resultado un modelo con 10 ítems. La ECI presentó índices de bondad de ajuste muy aceptables, de acuerdo con los índices seleccionados como criterios $\left(X^{2}=41.977, p=.112, X^{2} / g l=1.312\right.$, $C F I=.990, G F I=.956, N F I=.959, R M S E A=.043)$. 
Se observa que entre los constructos existe una correlación de .81, entre el capital humano y el capital estructural; también existe una correlación entre capital humano y capital relacional de .75 y entre capital estructural y capital relacional, la correlación fue de .84 (ver Figura 1).

Tabla 4

Estadísticos de bondad de ajuste

\begin{tabular}{lll}
\hline Estadístico & Abreviatura & Criterio \\
Ajuste absoluto & $X^{2}$ & Significación $>.05$ \\
Chi-cuadrado & $X^{2} / \mathrm{gl}$ & Menor que 3 \\
Razón Chi-cuadrado/grados de & & \\
Libertad & CFI & $\geq .95$ \\
$\begin{array}{l}\text { Ajuste comparativo } \\
\text { Índice de bondad de ajuste }\end{array}$ & & \\
$\begin{array}{l}\text { Comparativo } \\
\text { Otros }\end{array}$ & GFI & $\geq .90$ \\
$\begin{array}{l}\text { Índice de bondad de ajuste } \\
\text { Raíz de residuo cuadrático promedio de } \\
\text { aproximación }\end{array}$ & RMSEA & $<.10$ \\
\hline
\end{tabular}

Fuente: Elaboración propia.

Confiabilidad con los reactivos finales

Después de realizar el análisis factorial, se calculó la confiabilidad del instrumento con los ítems finales, obteniendo un alfa de Cronbach de .912.

\section{Conclusión}

El artículo presentó la confiabilidad y la validez de constructo de CI para pymes de Monterrey, Nuevo León, México. La ECI fue dimensionada de acuerdo al sustento teórico de varios autores (Álvarez y Gonzáles, 2013; Bontis, 2002; Bontis y Richardson, 2000; Buenechea, 2017; Herremans et al., 2011; Marzo y Scarpino, 2016; Matricano, 2016; Mention y 
Bontis, 2013; Ordóñez, 2003; Petty y Guthrie, 2000; Reyes, 2011; Roos et al. 2001; Sagástegui, 2014; Viedma, 2001; Wee y Chua, 2016) en las siguientes dimensiones: capital humano, capital estructural y capital relacional. La ECI se conformó con 16 ítems. Al realizar el análisis factorial exploratorio con el SPSS, se encontró que se cumplió con todos los criterios establecidos (KMO, prueba de esfericidad de Bartlett, matriz de covarianza anti-imagen, comunalidades y varianza total explicada). En cuanto a la solución factorial rotada, 14 ítems se agruparon en los factores correspondientes y dos ítems presentaron cargas más altas en otro factor. A pesar de que CI7 tenía una carga factorial mayor en el factor capital estructural, se decidió dejarlo en el factor capital humano puesto que tiene una carga similar en este factor y es en el que estaba inicialmente. El ítem CI12 tenía una carga factorial mayor en el factor capital estructural, pero se decidió dejarlo en el factor capital relacional, puesto que tiene una carga similar en este factor y es en el que estaba inicialmente. La confiabilidad final para la ECI fue de .912 y fue muy similar a otros estudios realizados tanto en el constructo como en los factores (Cabrita y Bontis, 2008; Chahal y Bakshi, 2016).

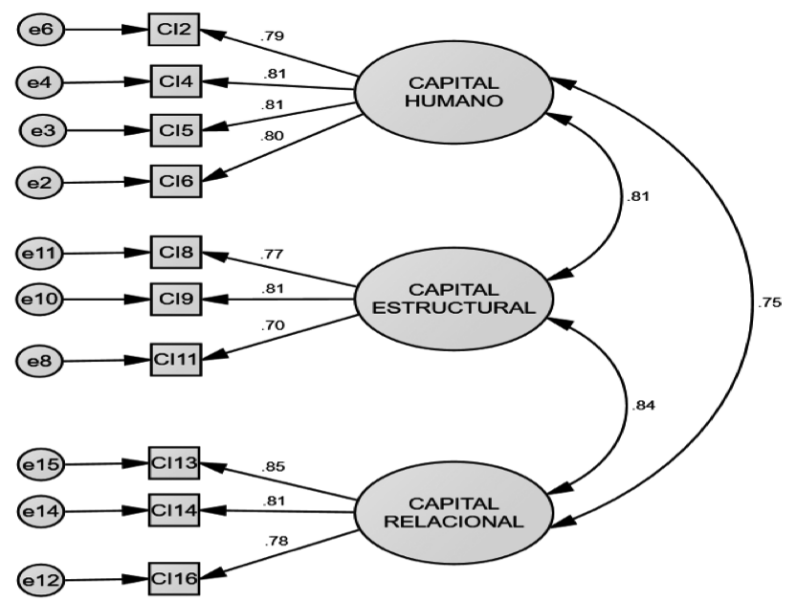

Figure 1. Parámetros estandarizados para el modelo capital intelectual.

Fuente: Elaboración propia

Al realizar el análisis factorial exploratorio con un modelo de ecuaciones estructurales, los resultados de los índices de ajuste del modelo propuesto muestran la aceptación del modelo con tres factores, los índices de bondad de ajuste fueron los siguientes: chi cuadrado $\left(X^{2}\right)$, razón chi cuadrado/grados de libertad $\left(X^{2} / g l\right)$, índice de bondad de ajuste comparativo $(C F I)$, 
índice de bondad de ajuste $(G F I)$ y raíz de residuo cuadrático (RMSEA). Los resultados de los índices de ajuste del modelo se cumplieron satisfactoriamente $\left(X^{2}=41.977, p=.112, X^{2} / g l=\right.$ $1.312, C F I=.990, G F I=.956, N F I=.959, R M S E A=.043)$, siendo estos índices mejores al compararse con los resultados de Chahal y Bakshi (2016), aunque estos autores realizaron la validación con un modelo de ecuaciones estructurales utilizando las dimensiones de los factores.

En conclusión, el análisis factorial exploratorio mostró que la ECI en las pymes de Monterrey, Nuevo León, México puede dimensionarse en los siguientes tres factores: capital humano, capital estructural y capital relacional. Al realizar la validez de constructo a través del modelo de ecuaciones estructurales, se corroboró la validez de la ECI, ya que todos los índices de bondad de ajuste seleccionados se cumplieron.

Este estudio tiene sus implicaciones en el sector empresarial, ya que contribuye a la validación de una escala para medir el CI en las pymes. Algunos autores (Dumay, 2014; Durst y Edvardsson, 2012; Galabova, 2014; Guthrie et al. 2012; Marzo y Scarpino, 2016; Mouritsen, 2006; ) recomiendan la realización de estudios del CI en las pymes desde un punto de vista no contable.

Para futuras investigaciones se recomienda probar la ECI en otros estados de México o países para comparar los resultados con este estudio.

Dentro de las limitaciones de este estudio, está el hecho de que no se realizó una discriminación y validación de los reactivos, utilizando diversas pruebas de piloteo con muestras grandes.

\section{Referencias}

Álvarez, C. N. y Gonzales, Z. G. (2013). Determinación de las clases de capital intelectual que afectan la correcta gestión de las pymes manufactureras metalmecánicos y servicios en la ciudad de Guayaquil. Recuperado de https://dspace.ups.edu.ec/bitstream/ 123456789/5300/1/UPS-GT000464.pdf

Aramburu, N., Sáenz J. y Blanco, C. E. (2015). Structural capital, innovation capability, and company performance in technology-based Colombian firms. Cuadernos de Gestión, 15(1), 39-60. doi:10.5295/cdg.130427na

Berezinets, I., Garanina, T. e Ilina, Y. (2016). Intellectual capital of a board of directors and its elements: introduction to the concepts. Journal of Intellectual Capital, 17(4), 632-653. DOI: 10.1108/JIC-01-2016-0003

Bontis, N. (1998) Intellectual capital: an exploratory study that develops measures and models. Management Decision, 36(2), 63-76.

Bontis, N. (2002). National intellectual capital index: Intellectual capital development in the Arab Region. Ontario: Institute for Intellectual Capital Research.

Bontis, N. Chua, W. y Richardson, S. (2000). Intellectual capital and business performance in Malaysian industries. Journal of Intellectual Capital, 1(1), 85-100. DOI: 10.1108/ 14691930010324188

Bontis, N., Janosevic, S. y Dzenopoljac, V. (2015). Intellectual capital in Serbia's hotel industry. International Journal of Contemporary Hospitality Management, 27(6), 1365-1384. DOI: 10.1108/IJCHM-12-2013-0541 
Buenechea, M. (2017). Structured literature review about intellectual capital and innovation. Journal of Intellectual Capital, 18(2), 262-285. DOI: 10.1108/JIC-07-2016-0069

Cabrita, M. D. R. y Bontis, N. (2008). Intellectual capital and business performance in the Portuguese banking industry. International Journal of Technology Management, 43(1-3), 212-237. DOI: 10.1504/IJTM.2008.019416

Cassol, A., Reis, C., Santos, A. y Lima, R. (2016). A administração estratégica do capital intelectual: um modelo baseado na capacidade absortiva para potencializar inovação. Revista Ibero Americana de Estratégia, 15(1), 27- 43. DOI: $10.5585 /$ riae.v5il.2161

Chahal, H. y Bakshi, P. (2016). Measurement of intellectual capital in the Indian banking sector. Vikalpa, 41(1), 61-73. DOI: $10.1177 / 0256090916629253$

Cordazzo, M. (2005). IC statement vs environmental and social reports: an empirical analysis of their convergences in the Italian context. Journal of Intellectual Capital, 6(3), 441-464. DOI 10.1108/14691930510611166

Curado, C., Henriques, L. y Bontis, N. (2011). Intellectual capital disclosure payback. Management Decision, 49(78), 1080-1098 DOI 10.1108/00251741111151154

Denicolai, S., Ramusino, E. C. y Sotti, F. (2015). The impact of intangibles on firm growth. Technology Analysis \& Strategic Management, 27(2), 219-236. DOI: 10.1080/09537325 .2014.959484

Dumay, J. (2014). 15 years of the journal of intellectual capital and counting: a manifesto for transformational IC research. Journal of Intellectual Capital, 15(1), 2-37. DOI: 10 .1108/JIC-09-2013-0098

Durst, S. y Edvardsson, I. R. (2012). Knowledge management in SMEs: a literature review. Journal of Knowledge Management, 16(6), 879-903. DOI: 10.1108/ 13673271211276173

Edvinsson, L. y Malone, M. S. (1997). Intellectual capital: Realising your company's true value by finding its hidden brainpower. New York: Harper Collins.

Edvinsson, L. y Malone, M. S. (1999). El capital intelectual. Barcelona: Gestión 2000.

Edvinsson, L., y Malone, M. S. (2003). Capital intelectual. Barcelona: Gestión 2000.

Euroforum. (1998). Medición del capital intelectual. Modelo intelect. Madrid: Euroforum. Recuperado de http:// gestiondelconocimiento.com/modelo_modelo_intelec.htm

Firer, S. (2005). Using intellectual capital as a success strategy in South Africa. Southern African Business Review, 9(1), 1-20.

Galabova, L. (2014). Recognition and management of intangibles by Bulgarian entrepreneurial firms. Journal of Intellectual Capital, 15(3), 376-391. DOI: 10.1108/JIC-05-2014-0056

Gates, S. y Langevin, P. (2010). Human capital measures, strategy, and performance: HR managers' perceptions. Accounting, Auditing \& Accountability Journal, 23(1), 111-132. DOI: 10.1108/09513571011010628

Guthrie, J., Ricceri, F. y Dumay, J. (2012). Reflections and projections: a decade of intellectual capital accounting research. The British Accounting Review, 44(2), 68-82. https: //doi.org/10.1016/j.bar.2012.03.004

Hair, J., Anderson, R., Tatham, R. y Black, W. (2007). Análisis multivariante (5 ed.). Madrid: Prentice Hall Iberia.

Hejazi, R., Ghanbari, M. y Alipour, M. (2016). Intellectual, human and structural capital effects on firm performance as measured by Tobin's Q". Knowledge and Process Management, 23(4), 259-273. https://doi.org/10.1002/ kpm.1529

Hernández, M. L. O., Moreno, M. B. P. y Arroyo, A. S. (2010). Análisis exploratorio de la implantación y uso de los Sistemas de Información sobre el Capital Intelectual en empresas castellano-leonesas: evidencia empírica e ideas para la reflexión. Revista Española de Financiación y Contabilidad, 39(145), 109-148.

Herremans, I. M., Isaac, R. G., Kline, T. J. y Nazari, J. A. (2011). Intellectual capital and uncertainty of knowledge: control by design of the management system. Journal of business ethics, 98(4), 627-640.

Janosevic, S. y Dzenopoljac, V. (2012). Impact of intellectual capital on financial performance of Serbian companies. Actual Problems of Economics, 133(7), 554-564.

Johanson, U., Mårtensson, M. y Skoog, M. (1999). Measuring and managing intangibles: eleven Swedish exploratory case studies. Paper presented at the International Symposium Measuring Reporting Intellectual Capital: Experiences, Issues, and Prospects, OECD, Amsterdam, June. 
Johnson, W.H.A. (1999). An integrative taxonomy of intellectual capital: Measuring the stock and flow of intellectual capital components in the firm. International Journal of Technology Management, 18(5-8), 562-575.

Jordão, R. V. D. y Novas, J. C. (2017). Knowledge management and intellectual capital in networks of small and medium-sized enterprises. Journal of Intellectual Capital, 18(3), 667-692. DOI 10.1108/JIC-11-2016-0120

Kemppilä, S. y Lönnqvist, A. (2003). Subjective productivity measurement. The Journal of American Academy of Business, 2(2), 531-537.

Keong, K. (2008). Intellectual capital: definitions, categorization and reporting models. Journal of Intellectual Capital, 9(4), 609-638. DOI: 10.1108/14691930810913186

Kujansivu, P. y Lönnqvist, A. (2007). Investigating the value and efficiency of intellectual capital. Journal of Intellectual Capital, 8(2), 272-287. DOI 10.1108/ 14691930710742844

Kweh, Q. L., Lu, W. M. y Wang, W. K. (2014). Dynamic efficiency: Intellectual capital in the Chinese non-life insurance firms. Journal of Knowledge Management, 18(5), 937-951. https://doi.org/10.1108/JKM-06-2014-0240

Lev, B. (2001). Intangibles. Management, measurement and reporting. Washington: Brookings Institution.

Marimuthu, M., Arokiasamy, L. e Ismail, M. (2009). Human capital development and its impact on firm performance: Evidence from developmental economics. Journal of International Social Research, 2(8), $265-272$.

Martínez, M. R. (2006). A procedure to design a structural and measurement model of intellectual capital: An exploratory study. Information \& Management, 43(5), 617-626.

Marzo, G. y Scarpino, E. (2016). Exploring intellectual capital management in SMEs: An in-depth Italian case study. Journal of Intellectual Capital, 17(1), 27-51. DOI 10.1108/ JIC-09-2015-0075

Matricano, D. (2016). The impact of intellectual capital on start-up expectations. Journal of Intellectual Capital, 17(4), 654-674. DOI 10.1108/JIC-04-2016-0040

Medina, A. S., González, A. M. y Pérez, E. H. (2007). El concepto del capital intelectual y sus dimensiones. Investigaciones Europeas de Dirección y Economía de la Empresa, 13(2), 97-111.

Mention, A. y Bontis, N. (2013). Intellectual capital and performance within the banking sector of Luxembourg and Belgium. Journal of Intellectual Capital, 14(2), 286-309. DOI 10.1108/14691931311323896

Mercado, P. (2016). Validez inicial de una escala del capital intelectual en universidades. Universitas Pyschologica, 2(15), 109-119. doi:10.11144/Javeriana.upsy15-2.viem

Mertens, L. (1996). Competencia laboral: sistemas, surgimiento y modelos. Montevideo: Cinterfor.

Molodchik, M. A., Shakina, E. A. y Barajas, A. (2014). Metrics for the elements of intellectual capital in an economy driven by knowledge. Journal of Intellectual Capital, 15(2), 206-222. https://doi.org/10.1108/JC-082013-0091

Morris, C. (2015). An industry analysis of the power of human capital for corporate performance: Evidence from South Africa. South African Journal of Economic and Management Sciences, 18(4), 486-499. http://dx.doi. org/10.17159/2222-3436/2015/ V18N4a4

Mouritsen, J. (2006). Problematizing intellectual capital research: Ostensive versus performative IC. Accounting, Auditing \& Accountability Journal, 19(6), 820-841. DOI: 10.1108/09513570610709881

OECD. (2013). Supporting Investment in Knowledge Capital, Growth and Innovation. OECD Publishing.

Ordóñez, P. (2003). Intellectual capital reporting in Spain: A comparative review. Journal of Intellectual Capital, 4(1), 61-81. DOI: 10.1108/14691930310455397

Petty, R. y Guthrie, J. (2000). Intellectual capital literature review. Measurement, reporting and management. Journal of Intellectual Capital, 1(2), 155-176. https://doi.org/10 .1108/14691930010348731

Ramírez, R. y Constansa, S. (2009). Estudio del capital intelectual del personal docente del área de postgrado de la Facultad de las Ciencias de la Educación de la Universidad de Carabobo, para la gestión de maestría en educación inclusiva enmarcada en la estimulación temprana (Tesis de maestría). Universidad de Carabobo Valencia, Venezuela.

Reyes, M. (2011). La valoración del capital intelectual y su repercusión en el patrimonio de las empresas en el país (Tesis doctoral). Universidad Nacional de Trujillo, Trujillo, Perú. 
Roos, J., Roos, G., Dragonetti, N. C. y Edvinsson, L. (2001). Capital intelectual. El valor intangible de la empresa. Barcelona: Paidós empresa.

Saari, L. M. y Judge, T. A. (2004). Employee attitudes and job satisfaction. Human Resource Management, 43(4), 395-407. doi:10.1002/hrm.20032

Sagástegui, J. O. (2014). El capital intelectual y su incidencia en la valorización de los activos de las Mypes de la ciudad de Trujillo (Tesis doctoral). Universidad Nacional de Trujillo, Trujillo, Perú.

Sánchez, J. A. (2018). Modelo causal entre factores del modelo innovador, la reingeniería del negocio y el desempeño empresarial validado por directivos de pymes de Monterrey, Nuevo León (Tesis doctoral). Universidad de Montemorelos, Montemorelos, México.

Santos, A. C. (2012). Gestión del talento humano y del conocimiento. Bogotá: Ecoe.

Secundo, G., Dumay, J., Schiuma, G. y Passiante, G. (2016). Managing intellectual capital through a collective intelligence approach: an integrated framework for universities. Journal of Intellectual Capital, 17(2), 298-319. https://doi.org/10.1108/JIC-05-2015-0046

Schiuma, G. y Lerro, A. (2008). Intellectual capital and company's performance improvement. Measuring Business Excellence, 12(2), 3-9. DOI: 10.1108/ 13683040810881153

Serenko, A. y Bontis, N. (2004). Meta review of knowledge management and intellectual capital literature: Citation impact and research productivity rankings. Knowledge and Process Management, 11(3), 185-198. DOI: $10.1002 / \mathrm{kpm} .203$

Sullivan, P. H. (2000). Value driven intellectual capital: How to convert intangible corporate assets into market value. New Jersey: John Wiley \& Sons.

Ting, I. W. K. y Lean, H. H. (2009). Intellectual capital performance of financial institutions in Malaysia. Journal of Intellectual Capital, 10(4), 588-599. DOI: 10.1108/ 1469193091099666

Verbano, C. y Crema, M. (2016). Linking technology innovation strategy, intellectual capital and technology innovation performance in manufacturing SMEs. Technology Analysis \& Strategic Management, 28(5), 524-540. https://doi.org/10.1080/09537325.2015.1117066

Viedma, J. M. (2001). ICBS - Intellectual Capital Benchmarking System. Journal of Intellectual Capital, 2(2), 148-165. https://doi.org/10.1080/09537325.2015.1117066

Villena, F. y Souto, J. E. (2015). El impacto de los intangibles en el desempeño exportador de la Pyme manufacturera. Intangible Capital, 11, 13- 40. doi:10.3926/ ic.567

Wang, Z., Wang, N. y Liang, H. (2014). Knowledge sharing, intellectual capital,and firm performance. Management Decision, 52(2), 230-258. http://dx.doi.org/10.1108/MD-02-2013-0064

Wee, J. C. y Chua, A. Y. (2016). The communication of intellectual capital: The 'whys' and 'whats'. Journal of Intellectual Capital, 17(3), 414-438.

Youndt, M. A., Subramaniam, M. y Snell, S.A. (2004). Intellectual capital profiles: An examination of investments and returns. Journal of Management Studies, 41(2), 335-361. https://doi.org/10.1111/j.1467-6486.2004.00435.x

Yu, H. C., Wang, W. Y. y Chang, C. (2015). The stock market valuation of intellectual capital in the IT industry. Review of Quantitative Finance and Accounting, 45(2), 279-304. https://doi.org/10.1007/s11156-014-0437-5 